\title{
Metaheuristic optimization for parameter estimation in kinetic models of biological systems - recent development and future direction
}

\begin{abstract}
Background: Kinetic models with predictive ability are important to be used in industrial biotechnology. However, the most challenging task in kinetic modeling is parameter estimation, which can be addressed using metaheuristic optimization methods. The methods are utilized to minimize scalar distance between model output and experimental data. Due to highly nonlinear nature of biological systems and large number of kinetic parameters, parameter estimation becomes difficult and time consuming. Methods: This paper provides a review on recent development of parameter estimation methods, which has received increasing attention in the field of systems biology. The development of metaheuristic optimization methods is mostly focused in this review along with the development of largescale kinetic models. Results: Although a plethora of methods have been applied to the problem of parameter estimation, recent results show that most of the successful approaches are those based on hybrid methods and parallel strategies. In addition, the current software used for parameter estimation and the sources of biological data for kinetic modeling are also described in this review. This review also presents future direction in parameter estimation to meet current industrial demands, especially in systems biology applications. Conclusion: The development of numerous optimization methods for parameter estimation in kinetic models has brought much advancement in the application of systems biology. Currently, it seems that there are highly demanded for further development of efficient optimization methods to address the expansion of systems biology applications.
\end{abstract}

Keyword: Systems biology; Parameter estimation; Metaheuristic; Optimization; Kinetic model 